\title{
VAC. 18 - Identification of viral populations and genome stability of current Brazilian yellow fever vaccine strains using whole genome data
}

Amanda Araújo Serrão de Andrade ${ }^{1 *}$; André Elias Rodrigues Soares ${ }^{1}$; Luiz Gonzaga Paula de Almeida ${ }^{1}$; Cristiane Pinheiro Pestana ${ }^{2}$; Carolina Lessa Aquino ${ }^{2}$; Ana Teresa Ribeiro de Vaconcelos ${ }^{1}$; Marco Alberto Medeiros ${ }^{2}$.

1Laboratório Nacional de Computação Cientifica - LNCC;

2Fiocruz/Bio-Manguinhos.

Introduction: Yellow fever is a viral hemorrhagic fever caused by the mosquito-borne yellow fever flavivirus (YFV). There are currently two YFV attenuated vaccine strains that are used worldwide to prevent yellow fever, which were independently derived, 17DD (Bio-Manguinhos/ Fiocruz) and 17D-204 (World Health Organization). Sequencing and assembling the virus genomes of vaccine stocks can provide invaluable insights into viability and stability of vaccine strains, as maintaining stable vaccine strains is essential for vaccine mass production.

Objective: Sequencing and assembling the whole genomes of the YFV Brazilian vaccine strains, identifying viral populations and verifying their genome stability along the vaccine lineages.

Methodology: A total of 20 current YFV 17DD vaccine production lots, and two 17D vaccine lots were obtained and sequenced using Illumina's next-generation sequencing (NGS) platform. Their viral genomes were assembled into single genome scaffolds using the oldest vaccine viral ancestral available as reference. Every single-nucleotide polymorphism (SNP) was identified, filtered by quality and frequency, and classified as synonymous or non-synonymous. The viral population for each lot was identified, and then analyzed for their SNP positions and influence in general viral stability.

Results: The viral genomes for all vaccine lots sampled were sequenced and assembled, leading to the identification of six high-frequency SNPs between the 17DD vaccine lots and four between $17 \mathrm{D}$ lots. These SNPs were mainly transition mutations and synonymous, located at the beginning of the envelope sequence and in non-coding regions (NCR) of the viral genome. Other 45 high-frequency SNPs were found when comparing the 17DD and 17D lots, these SNPs were located at various points of the genome and are mainly synonymous.

Conclusion: The sampled 17DD and 17D strains present small differences in their nucleotide compositions, which could potentially lead to variations in immunogenicity and reactogenicity. However, the SNPs that were identified by this study are primarily localized in the NCR of the genome, not affecting the translated sequences. Our results indicate that while the wild-type genomic sequence is variable, the vaccine genomes are highly homogeneous, and corroborate the hypothesis that vaccines obtained from current vaccinal strains (17DD and 17D-204) are unlikely to rebuild diversity and pathogenicity from the virulent strains.

Keywords: Yellow Fever; Vaccine stability; 17DD 